$17^{\text {th }}$ International Congress of Metrology, 13008 (2015)

DOI: $10.1051 /$ metrology / 201513008

(C) Owned by the authors, published by EDP Sciences, 2015

\title{
Performance of Articulated Arm CMM using Virtual Spheres Gauge and geometry deviation analysis
}

\author{
Bruno Fagundes Ferreira ${ }^{1,2}$ and Antonio Piratelli-Filho ${ }^{3}$ \\ ${ }^{1}$ Faculdade de Tecnologia SENAI Ítalo Bologna, Goiânia, GO, Brazil \\ ${ }^{2}$ Pontifícia Universidade Católica de Goiás - PUC Goiás, Goiânia, GO, Brazil \\ ${ }^{3}$ Universidade de Brasília, UnB, Faculdade de Tecnologia, Depto. Engenharia Mecânica, Brasília, DF, Brazil
}

\begin{abstract}
This work proposes a new methodology for testing Articulated Arm Coordinate Measuring Machines (AACMM) performance using the Virtual Spheres Plate (VSP) gauge to evaluate the geometric characteristics during measurement. An aluminum $400 \mathrm{~mm}$ x $400 \mathrm{~mm}$ VSP having 16 pyramidal inserts with 4 conical holes at each was used to determine the point coordinates of the hole centers. The test was performed in a Romer Arm 100 AACMM using a $6 \mathrm{~mm}$ ruby-made spherical stylus with a rigid probe. The gauge was positioned in three different locations and positions in AACMM work volume and the points were determined three times in each combination. An algorithm was developed in MatLab softtware to calculate geometric figures with the points determined, based on least squares method. The geometric deviations of straightness and flatness were determined to characterize the performance of the machine in respect to this type of measurement. The results were compared with ones from GPad AACMM software and with NIST reference algorithm, proving suitable to AACMM evaluation. This test can give additional information about the performance and can be used to compare different measuring machines.
\end{abstract}

Key Words: Articulated Arm CMM; geometric characteristics; Virtual spheres gauge

Mots Clés: MMT Bras Articulé; deviation géométriques, Virtual Sphéres Étalon

\section{Introduction}

The introduction of new technologies in manufacturing has motivated changes in industrial processes, as the development of products using complex geometries and freeform surfaces to attain design needs. Measurement is essential to assure the quality and reliability of the products and the coordinate measurement technology is growing in application. This technology makes possible the integration of the measuring equipment with computer software to product development, inspection steps, virtual verification of tolerances, development of new products by reverse engineering, among others.

The measurement techniques involved in production processes provide data for decision-making aimed at improving quality, increasing productivity, reducing costs and boosting competitiveness in the global market [1]. The use of Coordinate Measuring Machines (CMM) makes possible the integration of the measurement equipment and the computer software to the product development cycle, with off-line programming of inspection steps, virtual verification of design tolerances, measurement of physical prototype, among other applications. A special model of CMM is the Articulated Arm CMM (AACMM) that works with a series of joints with a defined number of degrees of freedom and encoders angle meters. The machine software determine the three-dimensional position of a probe stylus from encoder readings and arm segment lengths [2].

The AACMM measurement uncertainty is high when compared to the conventional CMMs [3]. Vrhovec and Munich [4] listed the main types of errors and quantify their influence on the performance of a AACMM. Lin et al. [5] simulated the influence of systematic errors in encoders and how they propagate to the end of the measuring arm. The growing use of AACMMs has required the development of methods to check the performance and the most used is described in AISI/ASME B89.4.22-2004. This Standard requires two calibrated ball bars as measuring gauges. Recent works had proposed a new generation of gauges and methodologies of performance tests to evaluate geometric and dimensional characteristics of these measuring machines. 
The gauges used to carry out performance check of AACMMs can be one-dimensional, two-dimensional or three-dimensional. Santolaria et al. [6] presented a technique to improve the repeatability of articulated arm CMM in more than $50 \%$. The author evaluated the kinematic model of a pivoted arm MMC with six degrees of freedom by using a ball bar with carbon fiber and 15 ceramic balls with $22 \mathrm{~mm}$ diameter.

Cheng et al. [7] used a simple device to measure the probe deflection. Cuesta et al. [8] conducted a study based on repeatability and reproducitility (R\&R) tests with blocks and ring gauges. The same parameters were measured in a traditional MMC for results comparison and five trained operators were involved. Multiba et al. [9] presented a new method to test AACMM and compared with the B89.4.22-2004 and the VDI / VDE 2617 Standards. According to the authors, the increasing use of this type of equipment has been accompanied by a lack of laboratories certificates for calibration and performance.

Madruga et al. [10] carried out a study about the influence of the contact force on the performance of AACMMs. A sensor was developed to measure the contact force applied by an operator, and a standard ring was used to analyze the relationship between the contact force and the measurement errors. Madruga et al. [11] developed a new dimensional gauge to check AACMM performance. The proposed methodology distinguishes dimensional and geometric tolerances, while the current verification standards only consider the dimensional ones. A gauge with geometric features was presented, allowing the evaluation of the operator's measuring technique and machine accuracy.

A different model of gauge was developed to address the problem of AACMM performance, introducing the virtual geometric features. Piratelli-Filho and Lesnau [12] proposed a gauge using two groups of four holes used to determine points of two virtual spherical surfaces. A least squares computational algorithm creates the spheres and determines the distance between the center-to-center of the spheres. The proposed artifact was named Virtual Spheres Bar (VSB) and was used to evaluate the performance of an AACMM with rigid probe. The results were compared with the standard ANSI/ASME B89.4.222004 and an additional experiment applying fractional factorial design was carried out to minimize the number of trials.

An improvement of this gauge was considered by Piratelli-Filho et al. [13]. The authors developed a gauge built by an aluminum plate with pyramidal inserts, as presented in Fig. (1). The pyramidal inserts have 4 conical holes and the spherical stylus of the AACMM can determine only one point at each hole. The groups of four points are then fitted to virtual spheres and the distance center-to-center of spheres are calculated by the developed least squares algorithm. This gauge was named Virtual Spheres Plate (VSP) and proved useful for checking the performance of an AACMM with rigid probe. The authors argued that the VSP proved to be less error prone compared to conventional ball bar because of the absence of the error source associated with the spherecity of the physical spheres. Besides, the cost is reduced compared to the ball plate gauge. The performance was evaluated by determining the errors in the center-to-center distance among the virtual spheres.

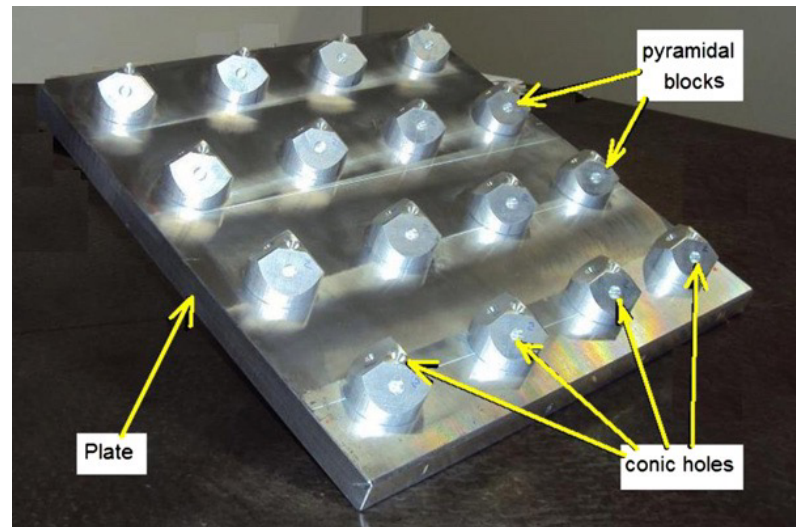

Figure 1. Virtual Spheres Plate [13].

This work proposes a new methodology for testing AACMM performance using the VSP gauge and evaluation of geometric characteristics [14]. The conical holes point coordinates of a $400 \mathrm{~mm} \times 400 \mathrm{~mm}$ VSP aluminum gauge were obtained by using a $6 \mathrm{~mm}$ rubymade spherical stylus with a rigid probe, using a Romer Arm 100 AACMM. The gauge was positioned in three different locations in AACMM work volume and the points were determined three times in each position. An algorithm was developed in MatLab to calculate the geometric elements with the points determined, based on least squares method to fit geometric figures and to determine the geometry deviations. The results were compared with the ones determined by the GPad machine software and proved suitable to AACMM evaluation and to comparison of different machines.

\section{Experimental procedure}

The experimental work was carried out with an articulated arm CMM (AACMM) Romer Arm 100, with a maximum arm reach of $2.5 \mathrm{~m}$ (spherical work volume). This CMM articulated arm has six encoders in a 2-2-2 configuration. The expanded volumetric measurement uncertainty $(95 \%)$ is $0.06 \mathrm{~mm}$, described in instrument calibration certificate. A rigid probe with spherical rubymade $6 \mathrm{~mm}$ diameter stylus was used to capture points in work volume, in conjunction with the software G-Pad ${ }^{\circledR}$.

The gauge "Virtual Spheres Plate (VSP)" was used to carry out tests in AACMM. This gauge has 16 groups of conical holes to fit 16 virtual spheres to data points and it is presented in Fig. (04). The same spherical stylus having diameter $6 \mathrm{~mm}$ was used in AACMM probe to capture the points on the conical holes. The gauge was calibrated in a Mitutoyo Cantilever CMM with $300 \mathrm{~mm} \times$ $400 \mathrm{~mm} \times 300 \mathrm{~mm}$ work volume and had an expanded uncertainty $(95 \%)$ of $(1.2+\mathrm{L} / 600) \mu \mathrm{m}$ ( $\mathrm{L}$ in millimeter).

The measurements took place with the Virtual Spheres Plate (VSP) placed in three positioning and three locations of the AACMM work volume. There were adopted three positions, near the reference AACMM basis ( $0-20 \%$ of the reach of the articulated arm), at intermediate distance (20 to $80 \%$ ) and far from the basis (80 to $100 \%$ ). The VSP positioning was adopted as nearly 
$200 \mathrm{~mm}$ (close), $500 \mathrm{~mm}$ (intermediate) and $850 \mathrm{~mm}$ (far) in respect to the basis. There were selected three different locations to each positioning: (A) left, (B) right and $(C)$ behind the center of the AACMM. These placements are showed in Fig. (02).

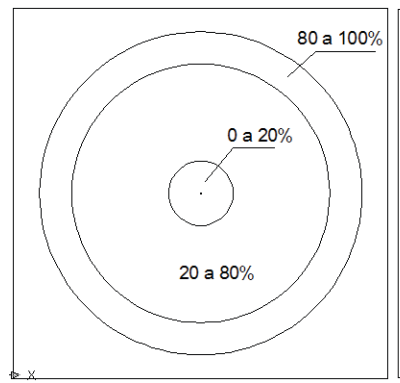

(a)

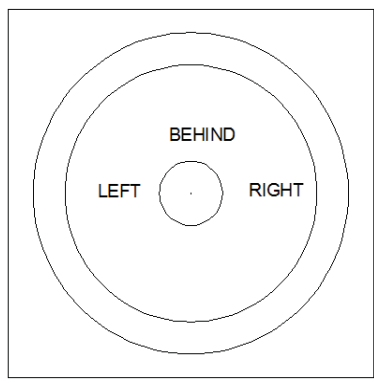

(b)
Figure 2. Positions (a) and locations (b) of the VSP gauge in respect to the AACMM basis.

For each position, a line with 16 points (diagonally in points 1, 6, 10 and 16) was measured and a plane was determined with all 64 points of VSP. The sequence of measurement was stablished by taking points according to the numbers marked in the VSP, from right to left and from the bottom to the top, as showed in Fig.(3). The data points were saved in files and imported using an algorithm built in MatLab. A 3D straight line and a plan were created at each position and location using the least squares method. The algorithm determines the distance among the points and the created geometric feature, stablishing the straightness and the flatness deviations.

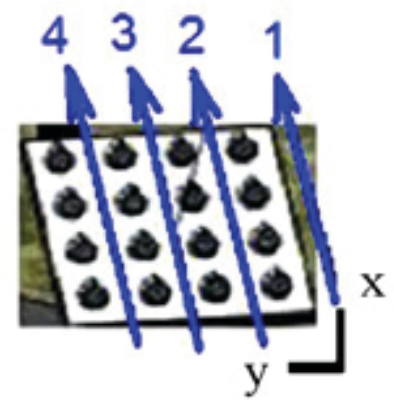

Figure 3. VSP (Points) [14]

The results of the proposed algorithm were compared with the machine G-PAD software for validation. The same points were used to fit the straight line and the plane and the straightness and flatness were determined with the software calculator.

Another comparison was performed with the reference algorithm developed by the National Institute of Standards and Technology (NIST). was used to validate the results obtained. This algorithm was based on the standard ISO B89.4.10 and has an extremely wide range of convergence [14]. The standard data provided by NIST in respect to the two-dimensional and three-dimensional circle, cone, cylinder, two-dimensional and threedimensional line, plane and sphere were used. These data were imported and considered as input to the developed algorithm, with the AACMM evaluation results compared to the results generated from the reference NIST algorithm $[15,16]$. Thus, the set of points extracted from NIST algorithm on internet [16] was implemented in the developed algorithm to determine a 3D line and a plane. The results were compared with the NIST results.

\section{Results}

\subsection{Proposed test}

The proposed test was performed in approximately two hours. The results of straightness and flatness are presented in Table (1), by the means and standard deviations. The standard deviations were small compared to the means and at almost the same order of the machine expanded uncertainty, varying between 0.016 and .

The mean straightness deviations are presented in Fig. (4) and Fig. (5), in respect to the position and location at AACMM. As observed, there is a small difference in straightness and the highest deviation for position was found when the VSP was measured close to the machine reference system, equals to $11.030 \mathrm{~mm}$. For location, the highest straightness deviation was found at left of the reference system, equal to $10.990 \mathrm{~mm}$. For flatness, it was observed a deviation of $8.540 \mathrm{~mm}$ at far distance from AACMM center (position), and $8.470 \mathrm{~mm}$ for behind location, the highest values.

Table 1. Mean and standard deviation of the straightness and flatness deviations determined.

\begin{tabular}{|c|c|c|c|c|c|c|c|}
\hline \multicolumn{2}{|c|}{} & \multicolumn{3}{|c|}{ Position } & \multicolumn{3}{c|}{ Location } \\
\cline { 3 - 8 } \multicolumn{2}{|c|}{ Line } & 1 & 2 & 3 & 1 & 2 & 3 \\
\hline \multirow{3}{*}{ Plane } & $\begin{array}{c}\text { Mean } \\
\text { (mm) }\end{array}$ & 11.026 & 10.834 & 10.928 & 10.971 & 10.993 & 10.825 \\
\cline { 2 - 8 } & $\begin{array}{c}\text { Std. } \\
\text { dev. } \\
(\mathrm{mm})\end{array}$ & 0.062 & 0.016 & 0.026 & 0.021 & 0.050 & 0.020 \\
& $\begin{array}{c}\text { Mean } \\
(\mathrm{mm})\end{array}$ & 8.436 & 8.409 & 8.540 & 8.447 & 8.464 & 8.473 \\
\cline { 2 - 8 } & $\begin{array}{c}\text { Std. } \\
\text { dev. } \\
(\mathrm{mm})\end{array}$ & 0.069 & 0.049 & 0.050 & 0.112 & 0.016 & 0.103 \\
\hline
\end{tabular}

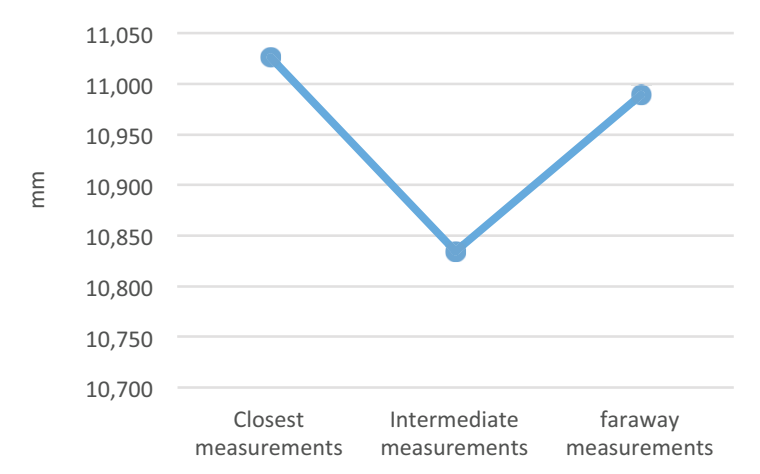

Figure 4. Mean straightness deviations position of the gauge

In respect to the orientation of the VSP gauge, it was found a small difference in straightness when the measurements were performed at left and at right of the VSP location in respect to the AACMM basis, as shown in Fig. (5). As the fitted geometry was as a virtual structure with points far from each other, it was obtained high values of the distance between the points and the line created, straightness, enabling better analysis. 


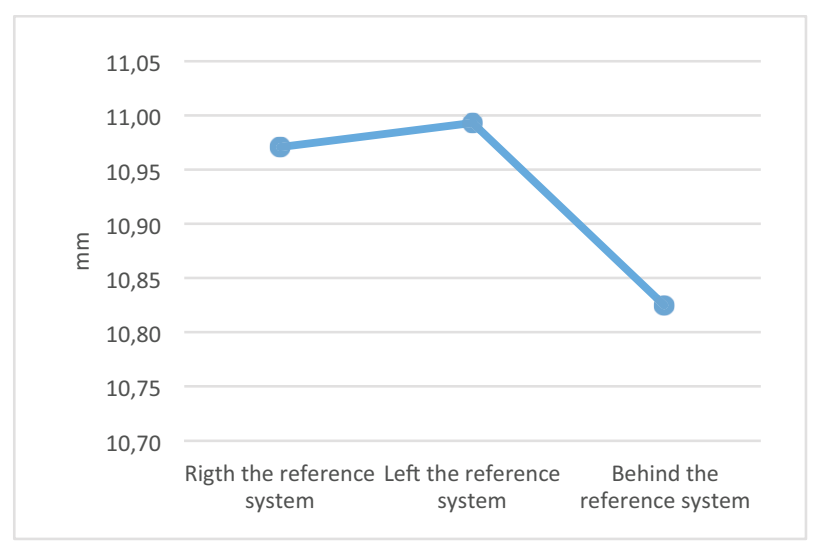

Figure 5. Mean straightness deviations according to the gauge location.

The flatness deviations are presented in Fig. (6) and (7). In respect to the measurement position, a small difference in flatness was observed, with the greatest error obtained when VSP was measured far from the AACMM reference system (80-100\%). It was also observed a small difference in measurement of flatness when measurements were carried out in the three locations, having the behind location the biggest values.

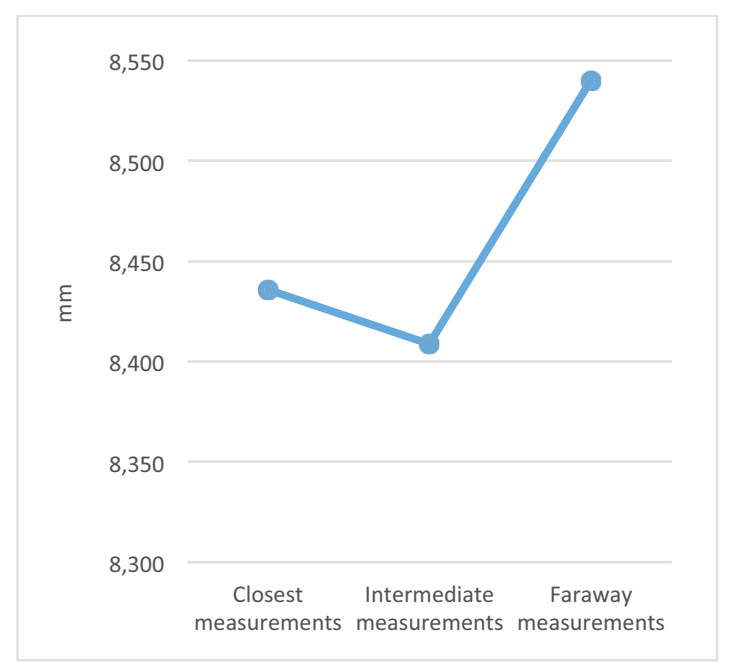

Figure 6. Flatness performance regarding the position of the gauge

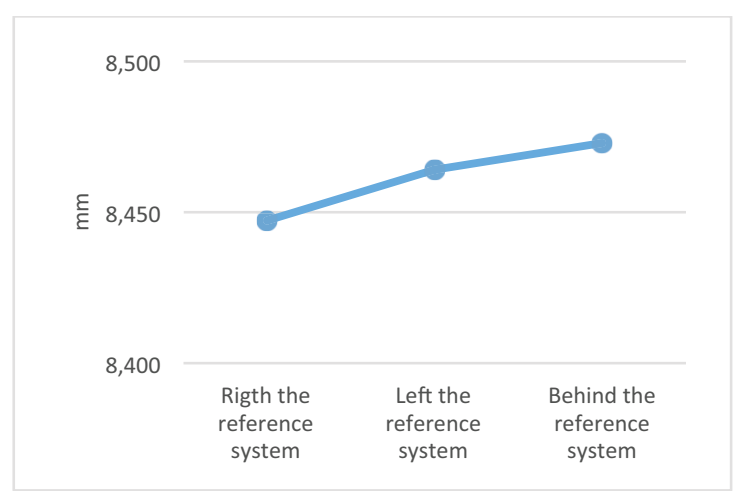

Figure 7. Flatness performance regarding the location of the gauge

3.2. Comparison with GPAD software
The proposed algorithm was compared with the results of the GPAD software. Nine lines were generated by the GPAD software, determining the deviations in respect to the fitted line and then the straightness deviations. The same nine lines were generated by the algorithm developed (by the coordinates of the 16 points measured for each of the lines), determining the orthogonal reference vector $(a, b, c)$ and the midpoint of the fitted line. Table 2 shows the results from these two methods and the difference in straightness. Figure (8) presents the comparison of the straightness. The maximum difference between the methods was observed to line 4, with difference of $0.348868 \mathrm{~mm}$. Six decimal places were considered as computational algorithms were used and compared.

Table 2. Analysis of results - Line 3D

\begin{tabular}{|c|c|c|c|c|c|}
\hline \multicolumn{6}{|c|}{ Line 3D } \\
\hline Line & & Straightness & $\mathrm{a}$ & $\mathrm{b}$ & $\mathrm{c}$ \\
\hline \multirow{3}{*}{1} & GPAD & 11.480000 & 0.612009 & 0.630339 & 0.477617 \\
\hline & Algorithm & 11.099445 & 0.612575 & 0.630280 & 0.476968 \\
\hline & Difference & 0.380555 & & & \\
\hline \multirow{3}{*}{2} & GPAD & 11.400000 & 0.591404 & 0.646174 & 0.482391 \\
\hline & Algorithm & 11.021370 & 0.593074 & 0.647991 & 0.477883 \\
\hline & Difference & 0.378630 & & & \\
\hline \multirow{3}{*}{3} & GPAD & 11.130000 & 0.661267 & 0.585860 & 0.468502 \\
\hline & Algorithm & 10.958766 & 0.660452 & 0.585136 & 0.470553 \\
\hline & Difference & 0.171234 & & & \\
\hline \multirow{3}{*}{4} & GPAD & 11.360000 & 0.593165 & 0.645693 & 0.480870 \\
\hline & Algorithm & 11.011132 & 0.594857 & 0.647527 & 0.476292 \\
\hline & Variation & 0.348868 & & & \\
\hline \multirow{3}{*}{5} & GPAD & 11.310000 & 0.572345 & 0.662288 & 0.483525 \\
\hline & Algorithm & 10.996060 & 0.574030 & 0.664230 & 0.478840 \\
\hline & Variation & 0.313940 & & & \\
\hline \multirow{3}{*}{6} & GPAD & 10.670000 & 0.512559 & 0.718399 & 0.470304 \\
\hline & Algorithm & 10.496115 & 0.511512 & 0.716927 & 0.473679 \\
\hline & Variation & 0.173885 & & & \\
\hline \multirow{3}{*}{7} & GPAD & 11.080000 & 0.619300 & 0.635809 & 0.460668 \\
\hline & Algorithm & 10.895389 & 0.618480 & 0.634964 & 0.462928 \\
\hline & Variation & 0.184611 & & & \\
\hline \multirow{3}{*}{8} & GPAD & 11.090000 & 0.700401 & 0.519989 & 0.488928 \\
\hline & Algorithm & 11.020098 & 0.701733 & 0.520974 & 0.485960 \\
\hline & Variation & 0.069902 & & & \\
\hline \multirow{3}{*}{9} & GPAD & 10.970000 & 0.681187 & 0.541106 & 0.493141 \\
\hline & Algorithm & 10.868328 & 0.682680 & 0.542288 & 0.489768 \\
\hline & Variation & 0.101672 & & & \\
\hline
\end{tabular}

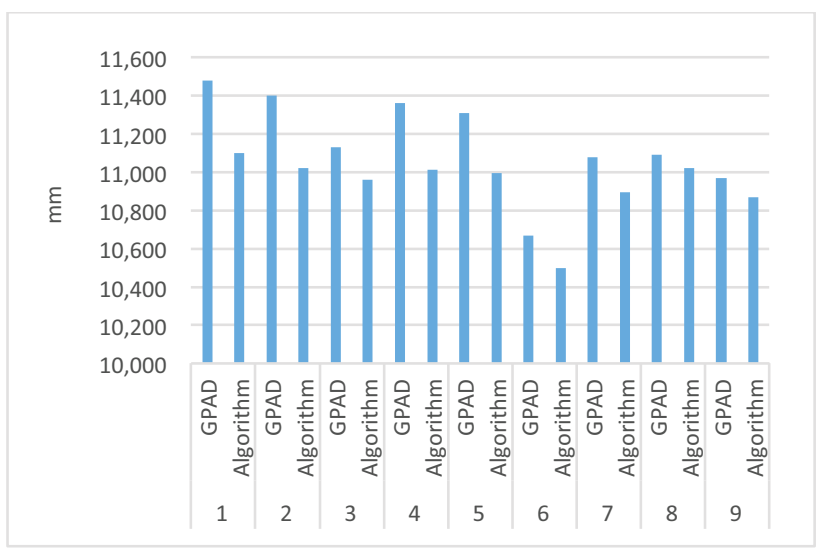

Figure 8. Straightness deviations of the proposed algorithm and GPAD. 
For flatness, nine plans were generated with the data points, determining the deviations between the points and the fitted plan and the flatness. Figure (9) presents the comparison of the results from developed algorithm and of the GPAD software. A variation up to $4 \mathrm{~mm}$ was observed between both methods, probably associated to the differences in the coordinates of reference vector found by each method.

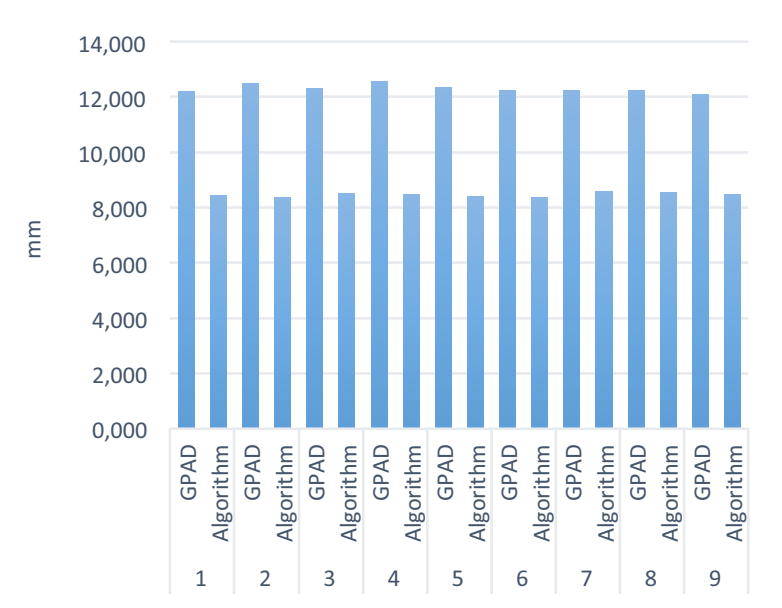

Figure 9. Flatness deviations of the proposed algorithm and GPAD.

\subsection{Comparison with NIST algorithm}

Additional comparison was performed against the NIST algorithm results. Table (3) presents the values of the components $(a, b, c)$ of the orientation vector for 4 straight lines and 2 planes, comparing the NIST and proposed algorithm. It was observed good agreement between the methods, since the values until the $11^{\text {th }}$ decimal place are equal.

Table 3. Comparison of NIST and proposed algorithm for calculated reference vector of the fitted line and plane.

\begin{tabular}{|c|c|c|c|c|}
\hline \multicolumn{5}{|c|}{ Line 3D } \\
\hline & & \multicolumn{3}{|c|}{ Vector } \\
\hline & & a & $\mathrm{b}$ & c \\
\hline \multirow{2}{*}{ Line 1} & \multirow{2}{*}{\begin{tabular}{|l|} 
Algorithm \\
NIST
\end{tabular}} & 0.00914926905 & \begin{tabular}{|l|}
0.00093280973 \\
\end{tabular} & 0.99995770948 \\
\hline & & 0.00914926905 & 0.00093280973 & 0.99995770948 \\
\hline \multirow{2}{*}{ Line 2} & \multirow{2}{*}{\begin{tabular}{|l|} 
Algorithm \\
NIST \\
\end{tabular}} & 0.20559869369 & 0.72866129088 & 0.65328546618 \\
\hline & & 0.20559869369 & 0.72866129088 & 0.65328546618 \\
\hline \multirow{2}{*}{ Line 3} & \multirow{2}{*}{$\begin{array}{l}\text { Algorithm } \\
\text { NIST }\end{array}$} & 0.01709426049 & 0.99981937792 & 0.00830649119 \\
\hline & & 0.01709426049 & \begin{tabular}{|l|}
0.99981937792 \\
\end{tabular} & 0.00830649119 \\
\hline \multirow{2}{*}{ Line 4} & \multirow{2}{*}{$\begin{array}{l}\text { Algorithm } \\
\text { NIST }\end{array}$} & 0.01419886685 & 0.99989861486 & 0.00107339865 \\
\hline & & 0.01419886685 & 0.99989861486 & 0.00107339865 \\
\hline \multicolumn{5}{|c|}{ Plane } \\
\hline & & \multicolumn{3}{|c|}{ Vector } \\
\hline & & $a$ & $\mathrm{~b}$ & $\mathrm{c}$ \\
\hline \multirow{2}{*}{$\begin{array}{c}\text { Plane } \\
1\end{array}$} & \multirow{2}{*}{$\begin{array}{l}\text { Algorithm } \\
\text { NIST }\end{array}$} & 0.73765919950 & 0.01530641891 & 0.67499971773 \\
\hline & & 0.73765919950 & 0.01530641891 & 0.67499971773 \\
\hline \multirow{2}{*}{$\begin{array}{c}\text { Plane } \\
2\end{array}$} & \multirow{2}{*}{$\begin{array}{l}\text { Algorithm } \\
\text { NIST }\end{array}$} & 0.20557246200 & 0.72884291570 & 0.65309108637 \\
\hline & & 0.20557246200 & \begin{tabular}{|l|}
0.72884291570 \\
\end{tabular} & 0.65309108637 \\
\hline
\end{tabular}

Figures (10) and (11) presents the comparison for straight line and plane, including the analysis of the center points. It is shown additionally that the center points have the same values until the $5^{\text {th }}$ decimal place.

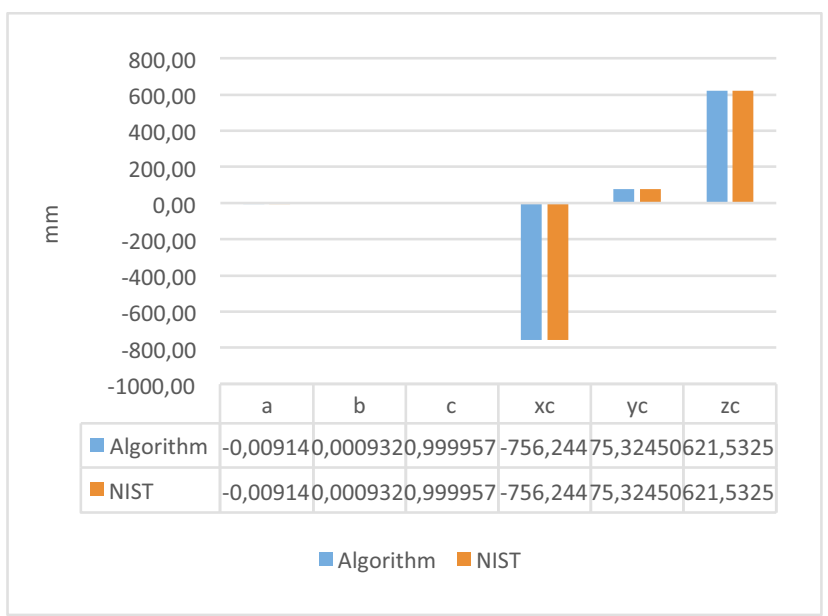

Figure 10. Comparison of unit vector and center of the fitted straight line: algorithm x NIST. The values of the $\mathrm{xc}, \mathrm{yc}$ and $\mathrm{zc}$ are in milimeters $(\mathrm{mm})$.

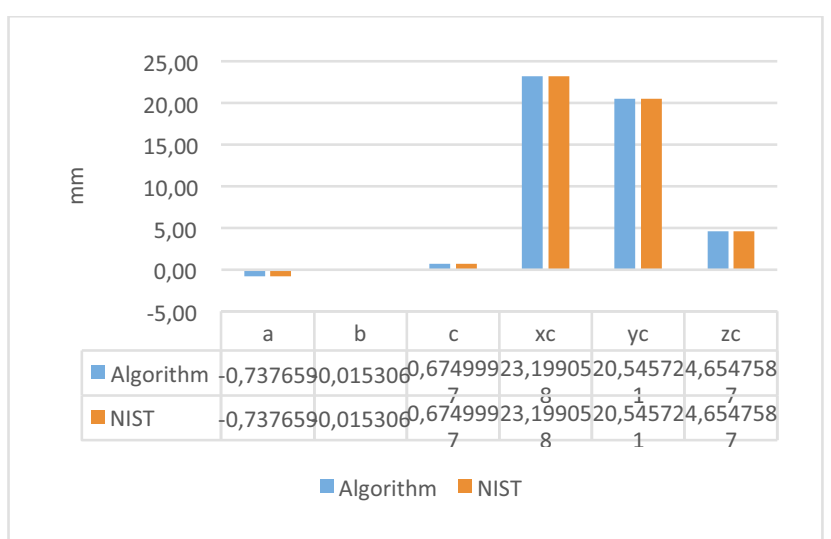

Figure 11. Comparison of unit vector and center of the fitted plane: algorithm x NIST. The values of the xc, yc and $\mathrm{zc}$ are in milimeters $(\mathrm{mm})$.

\section{Conclusion}

An algorithm for evaluating the AACMM performance by determining geometric tolerances of measured parts was developed and validated. The deviations of straightness and flatness were determined using a VSP gauge placed at three different positions and three different locations in machine work volume. The implemented approach proved suitable to complement the verification of performance of AACMMs. The algorithm was validated by comparing with data from NIST and with the results from GPAD AACMM software.

The results for the evaluated AACMM showed that the analysis for straightness and flatness allowed the determination of the smaller and bigger positions and locations to measure in the machine work volume. The standard deviations were small compared to the difference in means and an analysis of the means was sufficient to determine the differences.

Further research can be conducted to determine other geometries deviations as circularity, cilindricity and others. Application of statistical tools for analysis of the results can improve the quality of the performance test. 


\section{References}

[1] A.N.M. Santos. MsC Dissertation, Universidade de Brasília, DF, Brasil. (2012) (in portuguese)

[2] C.R.G. Lima. MsC Dissertation, Universidade Federal de Santa Catarina - UFSC. (2006) (in portuguese)

[3] L.A.R. Nerosky. MsC Dissertation, Universidade Federal de Santa Catarina - UFSC. (2001) (in portuguese)

[4] C.M. Vrhovec and M. Munich. Proc. IEEE/RSJ Int. Conf. Intelligent Robots and Systems (ICRA), Roma, Italy, pp. 697-702. (2007).

[5] S. Lin, P. Wang, Y. Fei. Int. J. Adv. Manuf. Technol., 879-886. (2005)

[6] J. Santolaria, J. Yague, J. Pastor. Precision Engineering, vol. 32, no. 4, pp. 251-268. (2008)

[7] W. Cheng, Y. Fei, L. Yu. Proc. SPIE 2010, $6^{\text {th }}$ Int. Symp. Prec. Eng. Measurements and Instrumentation, Hangzhou, China, p. 75445. (2010)

[8] E. Cuesta, B. Álvarez, J. Barreiro, D. Madruga. Proc. $4^{\text {th }}$ Manuf. Eng. Soc. Int. Conference. (2012)

[9] U. Mutilba, G. Kortaberria, A. Olarra, A. Guitérrez. J. Mech. Engineering and Automation, 602-608. (2013)

[10] D. Madruga, E. Cuesta, J. Barreiro, A. FernandezAbia. Sensors, Vol.13. (2013)

[11] D. Madruga, E. Cuesta, J. Alvarez, J. Barreiro. Meas. Sci. and Technology, Vol.25. (2014)

[12] A. Piratelli-Filho, A.R. Lesnau. Measurement, 43, 296-244. (2010)

[13] A. Piratelli-Filho, F.H.T. Fernandes, R.V. Arencibia. Precision Engineering, 36, 349-355. (2012)
[14] C. Diaz. Concept for an Algorithm Testing and Evaluation Program at NIST, National Institute of Standards and Technology. (1994)

[15] C.M. Shakarji. Least-Squares Fitting Algorithms of the NIST Algorithm Testing System, National Institute of Standards and Technology. (1998)

[16] http://www.nist.gov/pml/div683/grp01/cstalgorithmtesting.cfm, acessed in 01/07/2015.

\section{ACKNOWLEDGEMENTS}

The authors would like to acknowledge the Conselho Nacional de Desenvolvimento Científico e Tecnológico (CNPq), Brazil, and the Fundação de Apoio à Pesquisa do Distrito Federal (FAPDF), Brazil, for financing this work. 